\title{
Blind stock-taking
}

\section{For the past six months Nature Climate Change has been offering authors the option of double-blind peer review. Here we report on some preliminary findings from the trial.}

Double-blind reviewed manuscripts where both authors and referees remain anonymous - have accounted for between 12 and $30 \%$ of total monthly submissions to Nature Climate Change, with the six-month average around $22 \%$. This is somewhat lower than we might have expected on the basis of a reader survey run by Nature Geoscience in June 2012, where three-quarters of respondents agreed that double-blind peer review is a good idea.

As part of our monitoring effort, both journals involved in the trial (Nature Climate Change and Nature Geoscience) have been inviting all submitting authors to fill out a short survey to provide feedback on why they do or don't choose double-blind peer review. Completed surveys have been pooled to compensate for the relatively low number of respondents so far $(47 ; 12$ double blind, 35 traditional at the time of writing). The survey responses, although imperfect and self-selecting to some extent, indicated strong support for double-blind review in principle, with over $80 \%$ of respondents agreeing that it is a good (or very good) idea and 95\% supporting (or strongly supporting) the continuation of the trial. These numbers are encouraging, but pose the question: given such a high level of support in principle, why aren't the same numbers of authors choosing this option in practice?

The explanation seems to relate to levels of awareness about the trial, with about $60 \%$ of respondents unaware of the doubleblind option before submission. Of those who did not have previous knowledge of the trial, three-quarters said they would be more likely to choose the option if they knew about it before they started to write their paper. Reluctance to delay submission owing to the extra time it can take to retrospectively anonymize a manuscript accounts for this difference.

Preliminary records also indicate that referees do not seem significantly more reluctant to review double-blind manuscripts.

Interestingly, the perceived areas of bias that authors hoped double-blind review might mitigate were primarily concerned with author affiliations, influence and reputation. Gender and age were not perceived to be as significant as sources of bias. Two recurring concerns were expressed in the survey. First that double blind is most effective when mandatory, otherwise perceived bias might be introduced if referees assume those that select double blind are not from prestigious institutions, do not have an established reputation and so on. A number of authors also feel that their identities could be guessed anyway.

In summary, although uptake has been lower than we expected, early indications are that the trial is going well and that doubleblind review will increase in popularity as awareness of the option grows. An interesting question is whether we should indicate on published papers whether the review was single- or double-blind, but there are no plans to do this at present. In the next phase of monitoring we are aiming to investigate how often referees believe that they can identify authors, as well as their skill in doing so.

\section{Too little, too late?}

\section{Limited progress was made in Warsaw towards a universal agreement on action over climate change.}

The UN Climate Change Conference held in Warsaw in November 2013 had an inauspicious start. From the beginning, the conference was overshadowed by criticism of the host nation's attachment to coal, along with walkouts by pressure groups and backpedalling by Japan and Australia on previous climate commitments. And then in the middle of proceedings, much to the bemusement of delegates and commentators alike, conference president Marcin Korolec was replaced as Poland's Environment Minister by a proponent of hydraulic fracturing - 'fracking' - for shale-gas extraction.

However, some important decisions were made at the summit. For example, the Warsaw Framework for REDD+ has been established to help developing nations reduce their greenhouse gas emissions from deforestation and forest degradation. Although the details are yet to be hammered out, the framework includes financial rewards for countries that successfully reduce emissions by protecting their remaining forests.

Typhoon Haiyan struck the Philippines just before the meeting began, spotlighting the thorny issue of 'loss and damage'. Although rich, developed countries have pledged millions of dollars to the Adaptation Fund to support the poor nations vulnerable to climate change impacts, they are predictably chary of calls for international legal structures that might make them liable as a consequence of their past or present greenhouse gas emissions. Nevertheless, the Warsaw conference agreed to establish an international mechanism 'to provide the most vulnerable populations with better protection against loss and damage caused by both extreme weather events and slowonset events such as rising sea levels.'

The aim of the UN Framework Convention on Climate Change is for a final universal climate agreement to be signed in Paris in 2015. The new international agreement, effectively replacing the Kyoto Protocol, should come into force from 2020. But will it be so watered down through compromise as to be ineffective? Following the Warsaw meeting, Nicholas Stern, author of the 'Stern Review on the Economics of Climate Change' and chair of the Grantham Research Institute on Climate Change and the Environment and the Centre for Climate Change Economics and Policy at London School of Economics, released the following statement: "Although some progress has been made at this summit, the actions that have been agreed are simply inadequate when compared with the scale and urgency of the risks that the world faces from rising levels of greenhouse gases, and the dangers of irreversible impacts if there is delay."

We will find out soon enough how well international governments have been listening. 